\title{
Spatial Arrangement of Vasa Vasorum of the Human Great Saphenous Vein: Scanning Electron Microscopy and 3D-Morphometry of Vascular Corrosion Casts
}

\author{
B. Minnich,* A. Lametschwandtner,* D. Kachlik, ${ }^{* *}$ M. Setina, ${ }^{* * *}$ and J. Stingl $* *$ \\ * Vascular and Muscle Research Unit, Department of Organismic Biology, University of Salzburg, \\ Hellbrunnerstr. 34, 5020 Salzburg, Austria \\ ** Department of Anatomy, Third Medical Faculty of Charles University, Ruska 87, Prague 100 00, \\ Czech Republic \\ *** Department of Cardiovascular Surgery, Regional Hospital, Nemcove 54, Ceske Budejovice 370 \\ 04, Czech Republic
}

Recently, the spatial arrangement of vasa vasorum has been studied in arteries using micro-computer tomography $(\mu-\mathrm{CT})$ or scanning electron microscopy (SEM) of vascular corrosion casts $[1,2]$. As scanning electron microscopy (SEM) of microvascular corrosion casts allows to gain reliable data on the hierarchy of vessels, their exact branching patterns and branching angles, as well as the relationship between parent and daughter vessels in arterial branchings, respectively venous mergings, we recently combined this technique with 3D-morphometry and analyzed for the first time the $3 \mathrm{D}$-arrangement of vasa vasorum of the human great saphenous vein $(\mathrm{GSV})[3,4]$. Here we extend our work and ask which of the four optimality principles underlie the design of arterial and venous vasa vasorum bifurcations of the human GSV.

In five skeletonized and non-skeletonized segments of human GSVs, harvested during coronary bypass grafting, arterial feeders were exposed under the dissecting microscope, a glass cannula was introduced, and the vasa vasorum were rinsed and then casted with the polymerizing resin Mercox-Cl-2B. Stereopaired images (tilt angle: 6 degrees) were taken in the SEM (Cambridge 250), imported into a 3D-morphometry system [3] and diameters of arterial, venous and capillary vasa vasorum, intervascular and interbranching distances, and branching angles were measured. Data was used to calculate asymmetry ratios $(\alpha)$ and to test for given optimality principles [1].

Qualitative analysis of vascular casts in the SEM at low magnification showed that the wall of the GSV contained a multilayered vascular bed with the larger vessels running predominantly parallel to the longitudinal axis of the GSV. Venous vasa were more numerous and thicker than arterial vasa of the same branching generation (Fig. 1).

Quantitative analysis on GSV vasa vasorum revealed bifurcation indices $(\alpha)$ from $0.4-0.9$ in arterioles, $0.7-1.0$ in capillaries, and $0.4-1.0$ in venules. Interestingly, in venular bifurcations (mergings) the ratio of diameters of large (d1) and small daughter vessels (d2) to the diameters of their parent vessels $(\mathrm{d} 0)$, i.e. $\mathrm{d} 1 / \mathrm{d} 0$ respectively $\mathrm{d} 2 / \mathrm{d} 0$, was the same (0.6; mean). In arterioles this ratio was 0.9 (mean). While larger daughter vessels showed the same diameter ratio to the parent vessels in both, arterioles and venules $(0.6$; mean) this ratio was different in smaller daughter vessels (0.9 vs. 0.6 ; means) [2].

Testing indexes $\alpha$ and branching angles $\theta_{1}$ and $\theta_{2}$ of arterial, venous and capillary bifurcations respectively mergings revealed that bifurcations of the vasa vasorum of the human greater GSV are in favor of the principles of minimum pumping power and minimum vessel volume rather than of the principles of minimum vessel surface and minimum endothelial drag (Fig. 2). 


\section{References}

[1] M. Gössl et al. Anat. Rec. 272A (2003) 526 - 537.

[2] I.M. Bayer et al. Cell Tissue Res. 307 (2002) $303-313$.

[3] A. Lametschwandtner et al. Anat. Rec. 281A (2004) 1372 - 1382.

[4] B. Minnich et al. J. Microsc. 195 (1999) 23 - 33.

[5] The authors thank Mag. Bernhard Stöttinger for computations and data graphing.
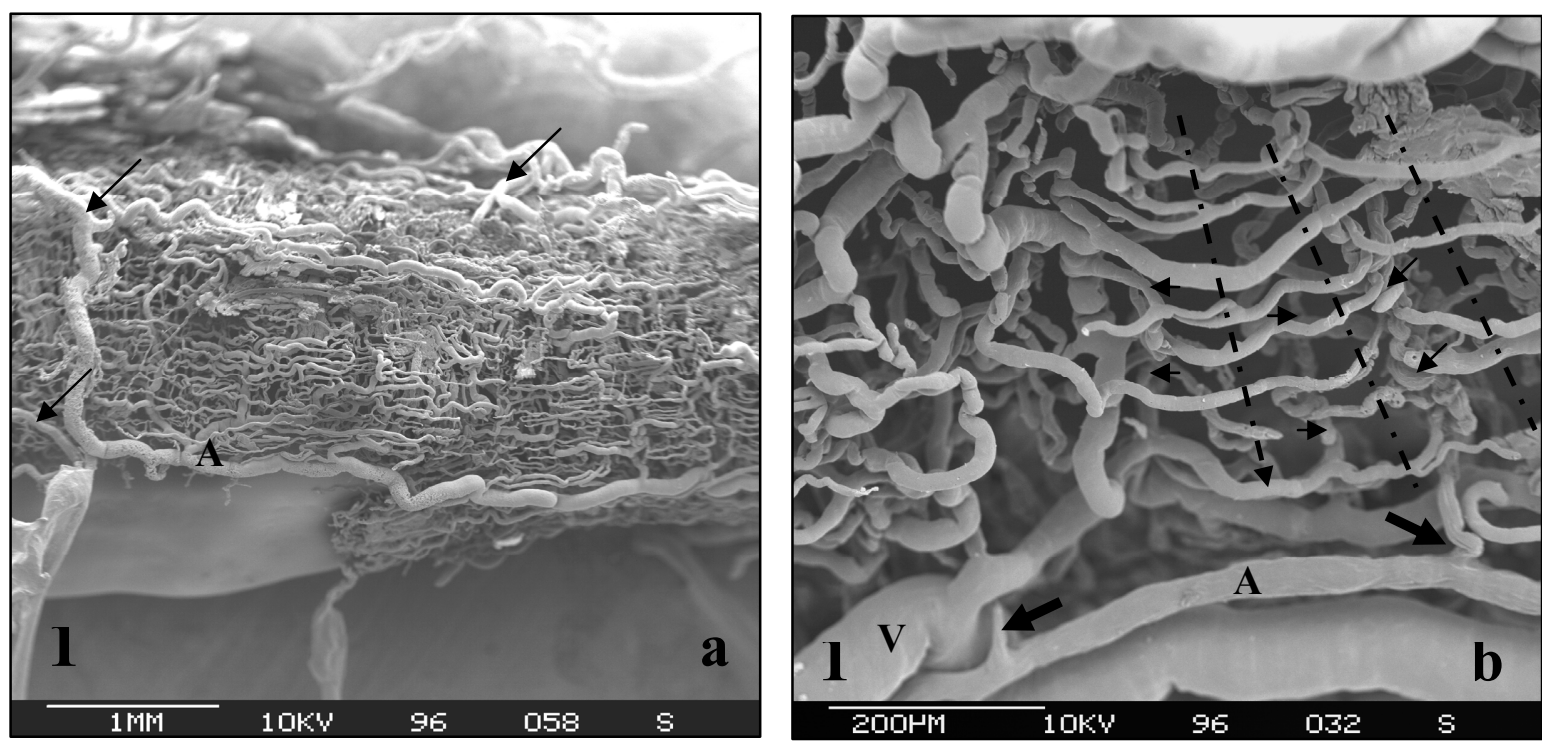

FIG. 1. Segment of the proximal portion of the human great saphenous vein (GSV) revealing the vasa vasorum from an adventitial view. Vascular corrosion cast. A. Overview. Note the prevailing longitudinal arrangement of the vasa. $\mathrm{A}=$ artery, $\mathrm{V}=$ vein. Arrows mark the feeding and/or draining vessels approaching the GSV every $0.5 \mathrm{~cm}-1.5 \mathrm{~cm}$. b. Detail. A thin artery (A) gives off arterioles (large arrows) supplying the dense network of vasa vasorum. Note the rather regularly spaced circularly running vessels (small arrow) outlining the location of the circular vascular smooth muscle cells (dashed lines).
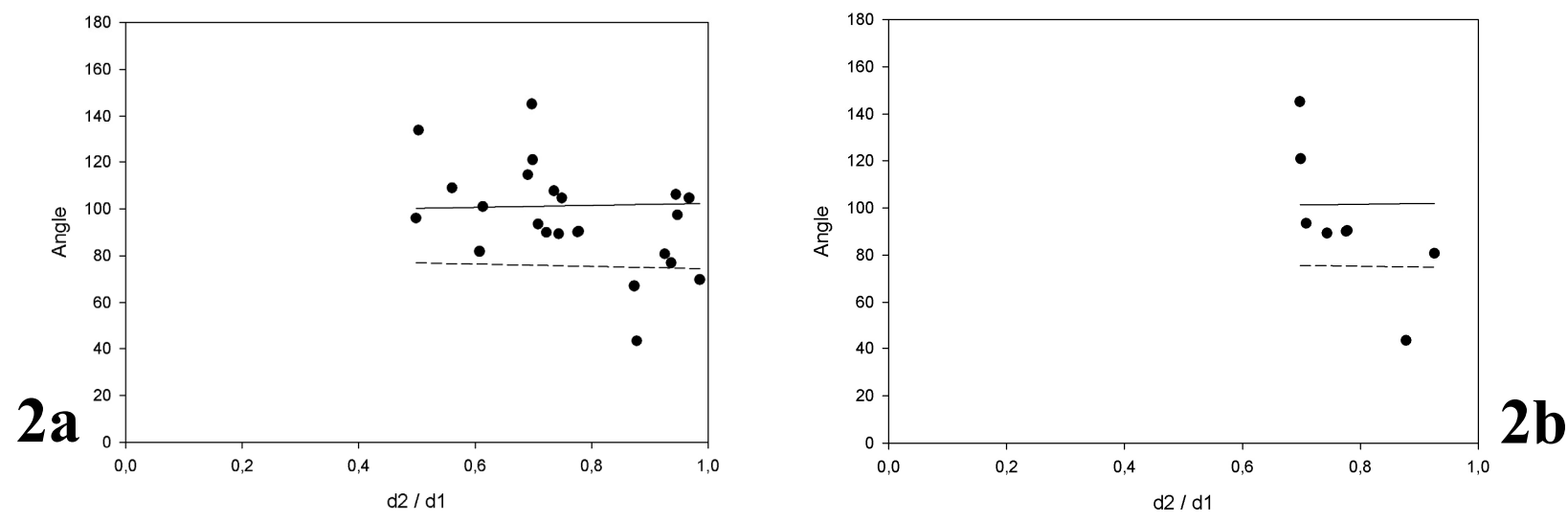

FIG. 2. The total angle between the two branches $(\theta 1+\theta 2)$ at bifurcations in the vasa vasorum of the GSV (a) venous side \& (b) arterial side, compared with the theoretical optima and plotted against the bifurcation index of each bifurcation. 